\title{
Measles surveillance system Evaluation in Benishangul Gumuz, Mandura woreda, Ethiopia in 2018
}

Dereje Zena ( $\sim$ derejezena@gmail.com )

https://orcid.org/0000-0002-4418-8899

kassahun Asires

Mandura woreda

Tigist Genetu

Bahir Dar hospital

Anemaw Asrat

Bahir Dar University

Research note

Keywords: Measles, Evaluation, Surveillance and Children

Posted Date: October 11th, 2019

DOI: https://doi.org/10.21203/rs.2.12615/v2

License: (c) (i) This work is licensed under a Creative Commons Attribution 4.0 International License.

Read Full License 


\section{Abstract}

Objective The main objective of this study is to Evaluate measles surveillance system in Benishangul Gumuz, Mandura woreda, Ethiopia in 2018. Result The weekly reporting rates of the health facilities over the past 52 weeks of 2018 were $>80 \%$ timeliness and completeness as show below by line graph for health posts. All the weekly surveillance reports were sent to the next level via report format and telephone. Mandura Woreda administration has made major progress in tackling the spread of measles. The overall structural set up of the surveillance system in creation of responsible unit /focal person of Public Health Emergency Management at each district and health facility level is good.

\section{Introduction}

Measles is a viral disease transmitted through the respiratory route and illness begins with fever, cough, and conjunctivitis followed by a characteristic rash. Most organ systems, with pneumonia accounting for most measles-associated morbidity and mortality due to its complications. The management of patients with measles includes provision of vitamin A (1). Measles can be prevented through vaccination. The only natural host of measles virus is human.

Measles can be occurred in all age groups. However, children younger than 5 years of age and adults older than 20 years of age are more likely to suffer from measles complications (2).

Measles is still commonly transmitted (endemic or large outbreaks) worldwide, including some countries in Europe, Asia, the Pacific, and Africa (2)

In Africa before the introduction of measles vaccination, measles was mostly a disease affecting young children, and more than 1 million cases were reported annually (3). But its incidence rate decreased by $85 \%$, from 841 cases in 2000 to 100 cases in 2015 per million populations in African region after the introduction of measles vaccine by WHO (4).

In Ethiopia, Measles has been one of the major causes of death and sickness of children. About 348 cases had been confirmed and 40 outbreaks reported in March 2017(5).

The surveillance system should be simple, flexible, acceptable and situation specific in response to an emergency in a given functioning system. Therefore, we want to evaluate the existing measles Surveillance system in Mandura woreda to know whether the surveillance system was well operated or not. Therefore, this evaluation addresses the following objectives:

1. To describe the best use of health resources and assure that systems operate effectively in Mandura woreda.

2. To know whether measles surveillance system is useful for community health and is achieving its objectives in the Woreda.

3. To evaluate some attributes of measles surveillance system in Mandura woreda. 


\section{Methods}

\subsection{Study setting}

Mandura woreda is one of the 7th woreda's of Metekel zone which is divided in to 20 kebele administrations (17 rural and 3 urban kebeles); The Woreda has 23 health posts, one health center, NonGovernmental Organization (NGO) medium clinic, four Private medium clinic and one prison clinic. The total population of Mandura is 55996(Male $=28110$ and Female $=27886$ ), from these about 1,719 are surviving infants, 9060 are under 5year children and 25,478 are under 15 years of age children. All 23 Health posts, 1 health center, 1 NGO medium clinic, 4 Private medium clinics and 1 Metekel prison clinic were reporting weekly measles surveillance reports.

\subsection{The design used}

To conduct measles surveillance system performance evaluation in Mandura woreda in 2018, the woreda Public Health Emergency Management (PHEM) and Health Management Information System (HMIS) reports were reviewed in addition to getting information through observational and in-depth interviews.

The performance of surveillance was evaluated according to United State (U.S) public health surveillance system guidelines

\subsection{Measles standard case definitions}

Measles is one of nationally immediately notifiable diseases that all health facilities (private and public) in Mandura woreda are expected to report to the woreda officers using the aforementioned case definitions.

The Ethiopian national Public Health Emergency Management and measles guideline was used for the case definitions (6)

Clinicians (internist, general practitioners, nurses, health officers) reported suspected measles cases with blood serum samples using case-based reporting form to Ethiopian Public Health Institute for laboratory analysis.

1.3.1. Suspected cases at community level: A community member should report any person with rash and fever to a health worker.

1.3.2. Clinical Suspected measles case: Any person with an acute illness characterized by:

- generalized, maculopapular rash lasting $\geq 3$ days;

- temperature $\geq 101^{\circ} \mathrm{F}$ or $38.3^{\circ} \mathrm{C}$; and

- cough, coryza, or conjunctivitis 
1.3.3. Epidemiological linked/Probable measles case: In the absence of a more likely diagnosis, an illness that meets the clinical description with:

- no epidemiologic linkage to a laboratory-confirmed measles case; and

- noncontributory or no measles laboratory testing.

1.3.4. Confirmed measles case: An acute febrile rash illness with:

- Isolation of measles virus from a clinical specimen; or

- Detection of measles-virus specific nucleic acid from a clinical specimen using polymerase chain reaction; or

- A significant rise in measles immunoglobulin $\mathrm{G}$ antibody using any evaluated and validated method; or

- A positive serologic test for measles immunoglobulin M antibody

\subsection{Indicators used}

Some useful indicators (attributes) were used to evaluate measles surveillance system performance in Mandura woreda such as: -

- Acceptability,

- Simplicity,

- Flexibility,

- Completeness/Data quality,

- Sensitivity,

- Positive Predictive Value,

- Representativeness,

- Timeliness,

- Stability and Validity

\subsection{Data source}

Different data sources such as; Mandura Woreda Measles surveillance database, annual reports, records and documents were used in addition to data gathered using in depth interview with standardized checklists and questionnaires.

\subsection{Collection procedure}

Standardized questionnaire was adapted from the World health Organization (WHO) Guideline based on the objectives of the evaluation. Interview was made to the surveillance officers and focal persons in the selected office for this evaluation purpose. Therefore, data were obtained from two ways of routine 
reporting system. These are PHEM and HMIS reports that are weekly and monthly reports respectively. PHEM data has been collected at health facilities and reported to woreda health offices weekly every Monday and on wards to the next level every next day of receiving the data. The HMIS reporting form integrated measles disease. The HMIS data are also collected at health facilities. However, these data were reported to woreda health office by hard copy monthly every 28 days.

\section{Results}

Any person with fever and maculopapular (non-vesicular) generalized rash and cough, coryza or conjunctivitis (red eyes) or any person in whom a clinician suspects measles in Mandura woreda were addressed under the existing Provider initiated (passive) population-based measles surveillance system.

Measles surveillance data is received from governmental health facilities, NGO health facilities missionary churches and private clinics. There was no shortage of reporting form in all health facilities and health office as we interview. The weekly reporting rates of the health facilities over the past 52 weeks of 2018 were $>80 \%$ timeliness and completeness as show below by line graph for health posts, but it was $100 \%$ health centers $100 \%$ All the weekly surveillance reports were sent to the next level via report format and telephone.

However, reporting through telephone is limited in health posts where there were no telephone services. In case of such setups, the HEWs use their personal mobile phones for emergency, for which they were complaining for refund, the other limitations telephone is affecting report objectivity

Among the 10 visited health facility, all of them have standard case definition of measles and the understanding of the cases definitions by the health extensions, $75 \%$ were good understanding, as they explaining us while we interviewing them at the time of the field visit. Concerning weekly PHEM reports format was there.

In this evaluation some useful indicators (attributes) were used to evaluate measles surveillance system performance in Mandura woreda. These indicators are presented as follow

4.1. Simplicity: In Madura woreda measles Case definition was understood at local \& Facility level but community measles case definitions were not well known due to different factors.

There was weekly report format at all health facility level. However, reporting is still paper-based at the health facility level and data reporting to the next level was by telephone calls and messages due to lack of computer \& internet services that induces report subjectivity and report delay.

4.2. Flexibility: In Mandura woreda, measles surveillance system was performed with adaptable manner to any without no difficulty in district.

4.3. Completeness/Data Quality: As we reviewed the annual report records, most required information was clearly recorded manually using standardized formats and log books and there were complete and 
valid data at the observed sites. Moreover, we checked data completeness/quality by checking the recorded and reported data (simple case counts) comparing with woreda FHEM reports. and asking the clarity of the case definition as indirect measure of data quality.

4.4. Acceptability. As we reviewed the surveillance report records, the concerned organizations have awareness on it and have a willingness to participate in the surveillance system. This was assured by completeness of report forms and timeliness of data reporting processes. But the Woreda didn't well engage for measles surveillance due to lack of multi-sectorial, health worker turns over, community cultural factor and poor geographical coverage as we interview \& identified by programmers.

4.5. Sensitivity: In Mandura, proportion of reported suspected measles cases in the woreda is $2(100 \%)$ as expected but Proportion of serum/ dried blood specimens arriving at lab within 3 days of being taken. This may be due to geographical settings, poor medical care infrastructure, and population working behavior and far to get health facilities. Even though, 1(50\%) blood specimen has been collected, the serological result feedback was not sent from the laboratory to the woreda.

4.6. Predictive Value Positive (PVP): We digit's get cases result received from the national measles laboratory department for father conformation. As a result, we couldn't measure the effect of PPV.

4.7. Representativeness: The health service coverage of Madura woreda is $96 \%$. The routine surveillance covers all governmental and some private health facility, and all population under surveillance in the catchment area. But the data we obtained cannot accurately reflect the characteristics of measles under surveillance in related to time, place, and person due to poor quality health care service provision i.e. one health center 57000 population, only geographical health coverages are covered by HP and HEWs. Therefore, the representativeness of the system in the woreda is likely to be low.

4.8. Timeliness. In Mandura Woreda reporting of measles cases to a public health agency and the time required for the identification of cases, outbreaks, or the effect of control and prevention measures including immediate control efforts, prevention of continued exposure and program planning was encouraging.

4.9. Stability: In Mandura Woreda, the surveillance system, the desired and actual amount of time required for the system to collect or receive data was stable.

\section{Discussion}

The Ethiopian Federal Ministry of Health and partners working in health has a plan to achieve at least $90 \%$ national coverage and $80 \%$ in every district with all vaccines by 2020 . The plan encompasses all components of immunization services: service delivery, vaccine supply, quality and logistics, disease surveillance and accelerated disease control, advocacy, social mobilization and communication and programme management. Therefore, we evaluate that case definitions understanding was found to be good among Healthcare providers including the health extension workers. 
The structure of data reporting flow from the lower to the upper level is well organized with unidirectional flow of data, with simple and defined role and responsibility of each reporting entities. However, the reporting flow has a number of obstacles such as inadequate infrastructure like transport, telephone, and computers for data management and analysis. These affected the overall generation of reports by the expected health facilities and make the surveillance system to relay on very limited reports validity rather than report consumption. These can make the surveillance system less useful to meet its objectives.

\section{Conclusion And Recommendation}

Mandura Woreda administration has made major progress in tackling the spread of measles.

The overall structural set up of the surveillance system in creation of responsible unit /focal person of PHEM at each district and health facility level is good.

Despite current achievements, Mandura Woreda is not on track to achieve the measles elimination goal of less than 1 case per million populations by 2020 with the current pace of elimination efforts.

Ethiopian $\mathrm{MOH}$ should make it a priority for resource allocation for each region to help Measles control programs and encourage Researches \& developments on Measles

Benishangul Gumuz RHB and Mandura Woreda health office should scale up the quality and implementation of all the measles elimination strategies, including the introduction of measles vaccine second dose (MCV2) in routine immunization services and should continue promoting local resource mobilization with sustained advocacy and tracking funds mobilization

\section{Limitation}

This evaluation system is limited only in Mandura woreda that didn't evaluate measles surveillance system in throughout the country.

\section{List Of Abbreviations}

HEWS--Health Extension workers

HMIS--Health Management Information System

HP----Health Professionals

$\mathrm{MOH}-$ Ministry of Health

NGO--Non-Governmental Organization

PHEM--Public health Emergency Management 
PVP---Predictive Value Positive

US-_-United State

WHO-World Health Organization

\section{Declarations}

\section{Ethics approval and consent to participate}

Ethical clearance and permission were obtained from Mandura health office through a formal Letter and written informed consent was obtained from each respondent before their interview.

\section{Consent for publication}

Not Applicable

\section{Availability of data and materials}

This research data is available at the hand of the corresponding Authors (derejezena@gmail.com). The Dara we gave will not be shared for others due to containing other than measles case reports

\section{Competing interests}

We declare that we do not have any competing interests.

\section{Funding}

No funding was given to support this study

\section{Authors' contributions}

The corresponding author (DZ, KA \& TG) was involved in the conception, design, drafted the manuscript and data analysis of the study. All authors (DZ, KA, TG, \& AA) were involved in analysis and interpretation of data.

\section{Acknowledgements}

Our deepest thank goes to Mandura woreda Health department higher officials for providing 2018 measles surveillance data for this evaluation. we would like to thank Bahir Dar University College of Medicine and Health Science Department of Epidemiology and Biostatistics for providing this opportunity for us.

\section{Authors' information}


Dereje Zena: He has BSc degree in Medical Laboratory technology and MPH in epidemiology gradating student from Bahir Dar university. He is working at Family Guidance association of Ethiopia, North West Branch

Email: derejezena@gmail.com

Kassahun Asires: He has BSc degree and MPH in epidemiology gradating student from Bahir Dar university. He is working at Mandura Worda, Ethiopia

Email: asiresk@gmail.com

Tigist Genetu: She has BSc degree in Medical laboratory and MPH in epidemiology gradating student from Bahir Dar university. She is working at Addisalem Hospital in Bahir Dar, Ethiopia

Email: tigistgenetu56@gmail.com

Anemaw Asrat: He has MPH in Epidemiology. Right now, he is an assistant professor working in Bahir Dar university as a lecturer

Email: anemawasrat@gmail.com

\section{References}

1. Nambulli S Sharp CR Acciardo AS Drexler JF Duprex WP Mapping the evolutionary trajectories of morbilliviruses: what, where and whither. Curr Opin Virol. 2016; 16: 95-105

2. Morbidity and mortality weekly report, Vol. 66 , no. 42, October 27,2017

3. Goodson JL, Masresha BG, Wannemuehler K, Uzicanin A, Cochi S. Changing epidemiology of measles in Africa. J Infect Dis. 2011;204(SUPPL. 1):205-14.

4. Patel MK, Gacic-dobo M, Strebel PM, Dabbagh A, Mulders MN. Progress Toward Regional Measles Elimination. Worldwide, 2000-2015. 2016;65(44):1228-33.

5. UNICEF, 17 May 2017

6. Accelerated Measles Control in Ethiopia: Integrated Measles SIAs Field Guide. Revised August 2010.

\section{Table}

Table 1: showing numbers of Measles case Surveillance reports in Mandura woreda in 2018

\begin{tabular}{|l|l|l|l|l|l|}
\hline Woreda & Expected & \multicolumn{4}{|c|}{ Number of Cases reported } \\
\cline { 2 - 6 } & $\begin{array}{l}\text { Case } \\
\text { based) }\end{array}$ & $\begin{array}{l}\text { Suspected Measles } \\
\text { DR }\end{array}$ & $\begin{array}{l}\text { NM Febrile rash } \\
\text { rate }\end{array}$ & $\begin{array}{l}\text { reported >=1 } \\
\text { case }\end{array}$ & $\begin{array}{l}\text { reported at least one } \\
\text { case }\end{array}$ \\
\hline Mandura 1 & 2 & 1 & 2 & 1 & \\
\hline
\end{tabular}




\section{Figures}

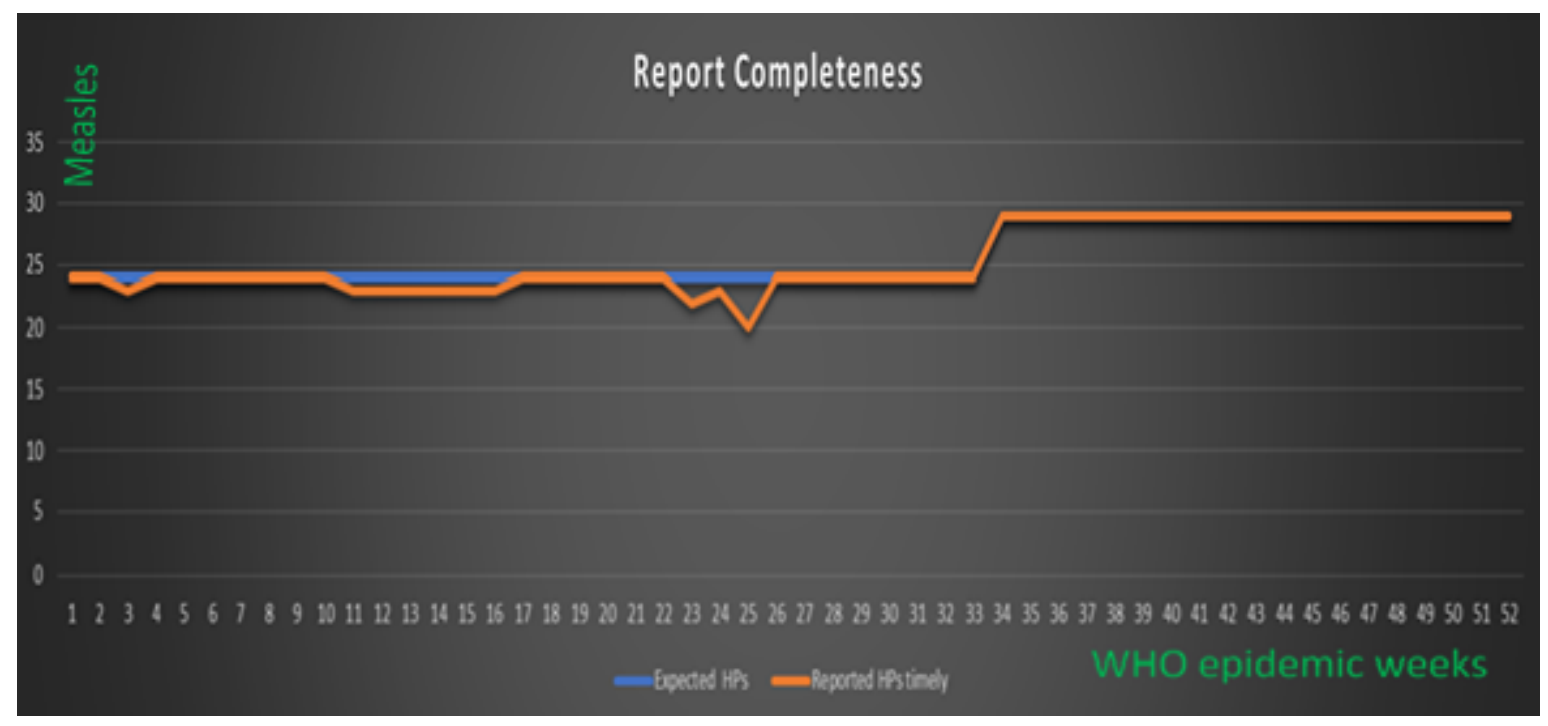

\section{Figure 1}

Graph 1: Line Graph showing Measles cases number report completes in Mandura woreda based on WHO epidemic weeks in 2018

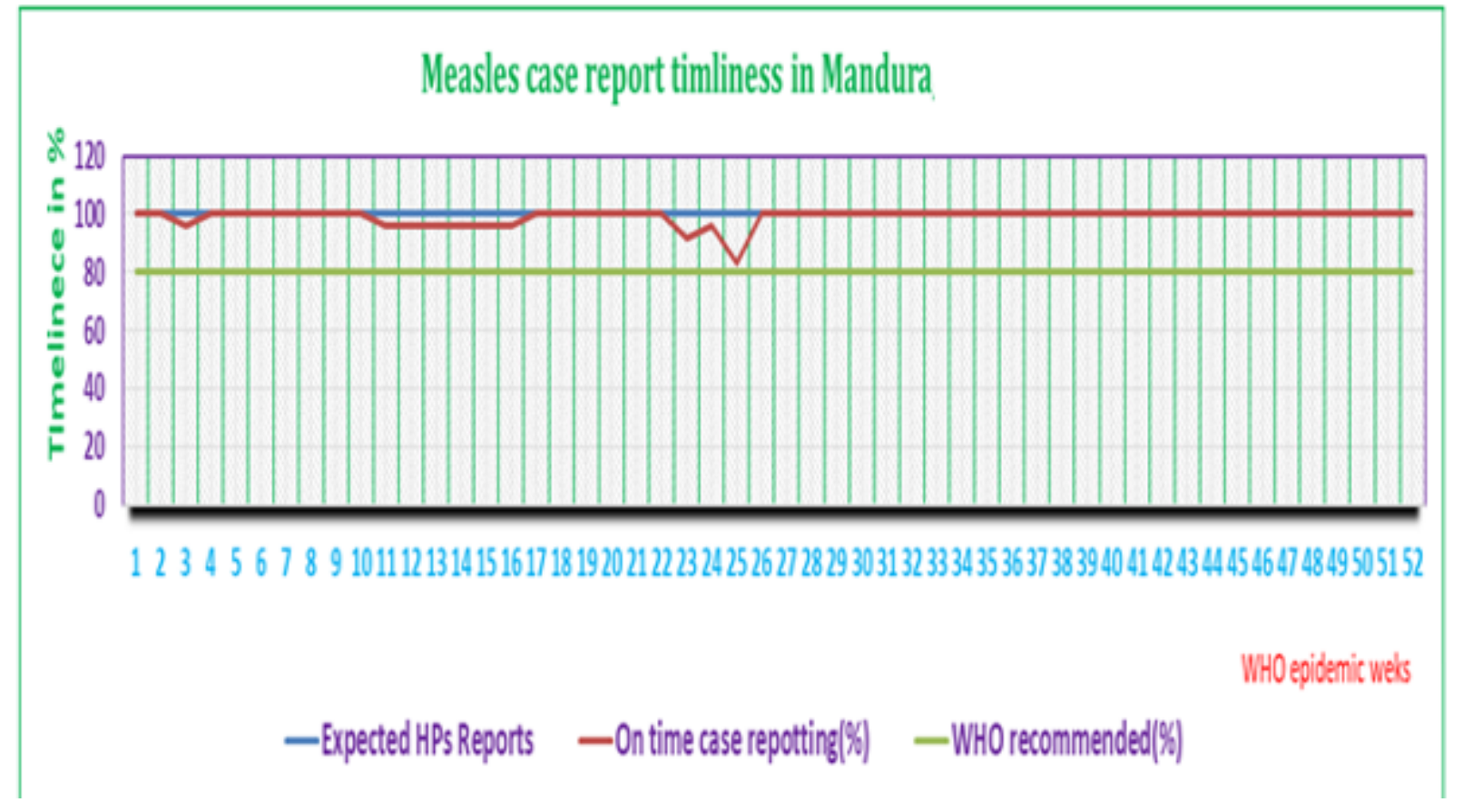

\section{Figure 2}

Graph 2: Line Graph showing proportion of Measles case report timeliness in Mandura woreda based on WHO epidemic weeks in 2018 